\title{
Negative dimensional approach for scalar two-loop three-point and three-loop two-point integrals
}

\author{
A. T. Suzuki and A. G. M. Schmidt \\ Universidade Estadual Paulista - Instituto de Física Teórica, R.Pamplona, 145, São Paulo SP, CEP 01405-900, Brazil
}

(June 24, 2018)

\begin{abstract}
The well-known $D$-dimensional Feynman integrals were shown, by Halliday and Ricotta, to be capable of undergoing analytic continuation into the domain of negative values for the dimension of space-time. Furthermore, this could be identified with Grassmannian integration in positive dimensions. From this possibility follows the concept of negative dimensional integration for loop integrals in field theories. Using this technique, we evaluate three two-loop three-point scalar integrals, with five and six massless propagators, with specific external kinematic configurations (two legs on-shell), and four three-loop two-point scalar integrals. These results are given for arbitrary exponents of propagators and dimension, in Euclidean space, and the particular cases compared to results published in the literature.
\end{abstract}

02.90+p, 11.15.Bt, 12.38.Bx

\section{INTRODUCTION.}

The study of quantum field theories in the perturbative regime can sometimes become a very challenging and arduous issue to deal with, especially when one needs to go on calculating multi-loop Feynman integrals. There are several techniques that have been developed and refined in the course of time, but usually the calculations are rather involved and analytic results are difficult to obtain. Depending on the approach one uses to tackle the problem, the obstacles to surmount can be more or less demanding. For example, if one uses the standard Feynman parametrization, the integral over momentum space is straightforward - in the sense that nowadays one can easily fint them out even tabulated in textbooks on quantum field theories - but the resulting parametric integrals that are left over become quite complicated and cumbersome to tackle, especially with increasing number of internal momenta in the loops. Of course, if one is clever enough, he/she may be able to overcome some of these hurdles and even perform really hard calculations [1.2].

There are, of course, other techniques which have been considered in the literature, e.g., integration by parts and Gegenbauer polynomial method in configuration space [9]; the Mellin-Barnes representation for massive propagators [10] to cite just a few of them [11, each one of those with its own strengths and weaknesses.

On the other hand, there is this novel technique known as negative dimensional integration method (NDIM), first devised by Halliday and Ricotta [3]. It has as its starting point the principle of analytic continuation, it has the remarkable property of being equivalent to Grassmannian integration in (positive) $D$-dimensional space 迎, it requires only the integration of Gaussian-like integrals and solving of linear algebraic equations and is suitable to deal with massless [5] or massive [6] diagrams in on- and off-shell regimes [7] and even to carry out light-cone gauge integrals, with added troublesome gauge-dependent poles [8].

Our aim in this work is to demonstrate the simplicity of NDIM calculating some scalar three-point integrals, with five and six massless propagators, at two-loop level and some two-point three-loop integrals also in the massless case. The outline for our work is as follows: in section II we present a detailed calculation of a two-loop integral in the NDIM approach; in section III we write down the results for the remaining two- and three-loop scalar integrals, while in section IV we give our concluding remarks about this work.

\section{TWO-LOOP THREE-POINT VERTEX WITH SIX MASSLESS PROPAGATORS.}

This computation is performed following just a few simple steps [5]. Fist of all, let us consider the Gaussian-like integral,

$$
\begin{aligned}
F & =\iint d^{D} r d^{D} q \exp \left[-\alpha q^{2}-\beta r^{2}-\gamma(q-r)^{2}-\delta(p+q)^{2}-\omega(q-k)^{2}-\theta(q-r-k)^{2}\right] \\
& =\left(\frac{\pi^{2}}{\lambda}\right)^{D / 2} \exp \left[-\frac{1}{\lambda}(\alpha \beta \delta+\alpha \gamma \delta+\beta \gamma \delta+\alpha \theta \delta) p^{2}\right]
\end{aligned}
$$


where the arguments in the exponential function of the integrand correspond to propagators in the diagram of Fig.1, with $k^{2}=t^{2}=0$ satisfying the on-shell condition. For the sake of compactness, we define

$$
\lambda=\alpha \beta+\alpha \gamma+\alpha \theta+\beta \gamma+\beta \delta+\gamma \delta+\theta \delta+\beta \omega+\gamma \omega+\theta \omega+\beta \theta .
$$

The parameters $(\alpha, \beta, \gamma, \delta, \omega, \theta)$ are quite general and arbitrary except that their real parts must be positive to make sure we have well-defined objects over the whole integration region.

Expanding the exponential in the second line of (1) in Taylor series and using the multinomial expansion for the argument of the exponential, we obtain,

$$
F=\pi^{D} \sum_{\{x, y, z=0\}}^{\infty} \frac{\left(-p^{2}\right)^{\Sigma x}(-\Sigma x-D / 2) ! \alpha^{a} \beta^{b} \gamma^{c} \delta^{d} \omega^{e} \theta^{f}}{x_{1} ! \cdots x_{4} ! y_{1} ! \cdots y_{9} ! z_{1} ! z_{2} !} \delta_{-\Sigma x-D / 2, \Sigma y+\Sigma z}
$$

where we have a fifteen-fold sum, with $\Sigma x=x_{1}+x_{2}+x_{3}+x_{4}$ and we define,

$$
\begin{aligned}
& a=x_{1}+x_{2}+x_{4}+y_{1}+y_{2}+y_{3}, \\
& b=x_{1}+x_{3}+y_{1}+y_{4}+y_{5}+y_{8}+z_{2}, \\
& c=x_{2}+x_{3}+y_{2}+y_{4}+y_{6}+y_{9}, \\
& d=x_{1}+x_{2}+x_{3}+x_{4}+y_{5}+y_{6}+y_{7}, \\
& e=y_{8}+y_{9}+z_{1}, \\
& f=x_{4}+y_{3}+y_{7}+z_{1}+z_{2} .
\end{aligned}
$$

On the other hand, taking the Taylor expansion of the exponential that is under the integration sign in (11), we have,

$$
F=\sum_{i, j, l, m, n, s=0}^{\infty} \frac{(-1)^{i+j+l+m+n+s}}{i ! j ! l ! m ! n ! s !} \alpha^{i} \beta^{j} \gamma^{l} \delta^{m} \omega^{n} \theta^{s} \mathcal{S}_{N D I M}^{1}
$$

where $\mathcal{S}_{N D I M}^{1}$ is the relevant integral in negative $D$, defined by

$$
\mathcal{S}_{N D I M}^{1}=\int d^{D} q d^{D} r\left(q^{2}\right)^{i}\left(r^{2}\right)^{j}(q-r)^{2 l}(q+p)^{2 m}(q-k)^{2 n}(q-r-k)^{2 s}
$$

Now comparing both expressions for the original integral $F$ we are led to the conclusion that, in order to have equality between these two expressions, the factor $\mathcal{S}_{N D I M}^{1}$ must be given by the multiple series,

$$
\mathcal{S}_{N D I M}^{1}=P_{1} \sum_{x, y, z=0}^{\infty} \frac{1}{x_{1} ! \cdots x_{4} ! y_{1} ! \cdots y_{9} ! z_{1} ! z_{2} !} \delta_{a, i} \delta_{b, j} \delta_{c, l} \delta_{d, m} \delta_{e, n} \delta_{f, s}
$$

with

$$
P_{1}=(-\pi)^{D}\left(p^{2}\right)^{\sigma} \Gamma(1+i) \Gamma(1+j) \Gamma(1+l) \Gamma(1+m) \Gamma(1+n) \Gamma(1+s) \Gamma\left(1-\sigma-\frac{1}{2} D\right)
$$

and for convenience we use the definition $\sigma=i+j+l+m+n+s+D$. The system we must solve has, therefore, fifteen "unknowns" (the indices $x, y, z)$ and only seven equations - six of which comes from comparing the exponents of $(\alpha, \beta, \cdots)$ and one from the multinomial expansion, namely, $\Sigma y+\Sigma z=-\Sigma x-D / 2$. So, such a system can only be solved in terms of some of these "unknowns", and we conclude, from the combinatorics, that there are $C_{7}^{15}=6435$ systems $(7 \times 7)$ of linear algebraic equations, i.e., there are 6435 different ways of choosing these "unknowns". Of this grand total possible solutions, 4113 yield trivial solutions and for this reason present no interest at all. Yet, the remaining 2322 solutions are quite a big number! Fortunately, not all the remnant non-vanishing solutions need to be considered in order to solve the Feynman integral. In fact, only linearly independent series will be needed. In our massless case and where there is only one non-vanishing external momentum, the argument of such series is unit. Since NDIM provides, in general, hypergeometric series (see, for example ref. [8] where we have double and triple series of such), we obtain in this case ${ }_{p} F_{q}(\cdots \mid 1)$, and from the theory of generalized hypergeometric functions 13 , 14], a linear combination of linearly independent series of this type has up to $p$ series.

Consider then, the solution where the indices $y_{2}, y_{3}, y_{4}, y_{5}, y_{7}, y_{8}, y_{9}, z_{2}$ are left undetermined. Solving this system we get, 


$$
\begin{aligned}
& x_{1}=-l+m+n-s+y_{2}+y_{3}+y_{4}-y_{5}-y_{8}+z_{2}, \\
& x_{2}=i+l+n+D / 2-y_{2}-y_{9}+y_{5}+y_{7}+z_{2}, \\
& x_{3}=j+l+s+D / 2-y_{4}-z_{2}, \\
& x_{4}=-n+s-y_{3}-y_{7}+y_{8}+y_{9}-z_{2}, \\
& y_{1}=-m-n-D / 2-y_{2}-y_{3}-y_{4}-z_{2}, \\
& y_{6}=-\sigma+m-y_{5}-y_{7} \\
& z_{1}=n-y_{8}-y_{9}
\end{aligned}
$$

which substituted in (6) yields an eight-fold summation. Seven of these can be rewritten in terms of gamma functions. The procedure is as follows: Firstly, the factorials must be converted into gamma functions and these into Pochhammer symbols, defined by,

$$
(n \mid k) \equiv(n)_{k}=\frac{\Gamma(n+k)}{\Gamma(n)}
$$

Secondly, use the properties,

$$
(n \mid j+k)=(n+j \mid k)(n \mid j), \quad(n \mid-k)=\frac{(-1)^{k}}{(1-n \mid k)},
$$

which follow from the definition above. Then, it is possible to rewrite some series in terms of Gaussian hypergeometric functions, which can, in general, be summed when its argument is unit 12 .

$$
{ }_{2} F_{1}(a, b ; c \mid 1)=\frac{\Gamma(c) \Gamma(c-a-b)}{\Gamma(c-a) \Gamma(c-b)} .
$$

Exception for this occurs when the parameters are such that $c-a-b=0$.

Therefore, if we rearrange these series conveniently, we can sum them, and applying this procedure for the series defined by indices $y_{4}, y_{3}, y_{2}, y_{8}, y_{9}, y_{7}, y_{5}$, they all can be summed out. The last series, defined by index $z_{2}$, however cannot be summed in this fashion since it is a ${ }_{3} F_{2}$ function, which is summable only when it is of Saalschützian type [13], and this is not our present case.

Many of the gamma functions that appear in this process simplify among themselves and in the end, what remains are fourteen gamma functions distributed in a fraction, i.e., seven in the numerator and seven in the denominator multiplying the $z_{2}$ series. This is the result in the negative dimension region and positive exponents $(i, j, l, m, n, s)$, see (4). Now we must be able to bring this result back, that is, analytically continue it, to our real physical world, that of positive $D$. This is carried out very easily: group the gamma functions in convenient Pochhammer symbols and use the second property (9).

Considering now the solution where $x_{1}, x_{2}, x_{3}, y_{1}, y_{5}, y_{6}, y_{9}, z_{2}$ are left undetermined, we get another ${ }_{3} F_{2}(a, b, c ; e, f \mid z)$ function. Let us recall that such functions have three singular points, namely $(0,1, \infty)$. Here, we are interested in solutions in the vicinity of $z=1$. N $\phi$ rlund [15] proved that when the difference between $e$ and $f$ is an integer, some of the series concide or are without meaning. This is precisely our case. This means that our final result for the Feynman integral shall exhibit only two linearly independent generalized hypergeometric functions of the type ${ }_{3} F_{2}$, instead of the usual three. We get, as a result, then

$$
\mathcal{S}_{N D I M}^{1}=\pi^{D}\left(p^{2}\right)^{\sigma}\left\{\mathbf{A}_{3} F_{2}\left(a_{1}, b_{1}, c_{1} ; e_{1}, f_{1} \mid 1\right)+\mathbf{B}_{3} F_{2}\left(a_{1}^{\prime}, b_{1}^{\prime}, c_{1}^{\prime} ; e_{1}^{\prime}, f_{1}^{\prime} \mid 1\right)\right\}
$$

where

$$
\begin{aligned}
\mathbf{A} & \equiv(-m \mid \sigma)(\sigma+D / 2 \mid-2 \sigma-D / 2)(-j \mid j+l+s+D / 2)(-l \mid j+l+s+D / 2) \\
& \times(-i-j-l-s-D / 2 \mid i)(j+l+s+D \mid-j-l-s-D / 2+m+n),
\end{aligned}
$$

and

$$
\begin{aligned}
\mathbf{B} & \equiv(-i \mid \sigma)(-m \mid \sigma)(\sigma+D / 2 \mid-2 \sigma-D / 2) \\
& \times(-j \mid-l-s-D / 2)(j+l+s+D \mid-j-D / 2)(-l-s \mid j+l+s+D / 2),
\end{aligned}
$$

where the set of parameters are defined as $\left(a_{1}, b_{1}, c_{1} ; e_{1}, f_{1}\right)=(-s, m+n+D / 2,-j-l-s-D / 2 ;-i-j-l-s-$ $D / 2,1-j-s-D / 2)$, and $\left(a_{1}^{\prime}, b_{1}^{\prime}, c_{1}^{\prime} ; e_{1}^{\prime}, f_{1}^{\prime}\right)=(-\sigma,-l,-j-l-s-D / 2 ;-l-s, 1+i-\sigma)$. Note that $\left(e_{1}-f_{1}\right)$ is 
an integer. Observe also that, in the special case where all the exponents are equal to minus one, the hypergeometric function ${ }_{3} F_{2}$ reduces to ${ }_{2} F_{1}$, which can be summed again, using (10). So, for this special case of negative unity exponents, the final result is expressed in terms of gamma functions only.

Let us point out here that our final result agrees with that given by Davydychev and Osland [1], i.e., meaning that equation (27) in ref. [1] disagrees with ours. The difference is in the $9 \zeta(4)$ factor in [1] which should correctly be read $9 \zeta(2)$. This gives the correct result for $i=j=l=m=n=s=-1$ and $D=4-2 \epsilon$.

\section{RESULTS FOR SOME TWO AND THREE-LOOP MASSLESS SCALAR INTEGRALS.}

The scalar integrals for the remaining diagrams (Fig.2-7) are, in negative $D$, given by (number superscripts 2-7 label the corresponding diagrams):

$$
\begin{gathered}
\mathcal{S}_{N D I M}^{2}=\int d^{D} q d^{D} r\left(r^{2}\right)^{i}\left(q^{2}\right)^{j}(p+r)^{2 l}(q+k)^{2 m}(p+r-q)^{2 n}, \\
\mathcal{S}_{N D I M}^{3}=\int d^{D} q d^{D} r\left(r^{2}\right)^{i}\left(q^{2}\right)^{j}(k-q-r)^{2 l}(k-r)^{2 m}(r+p)^{2 n}, \\
\mathcal{S}_{N D I M}^{4}=\int d^{D} q d^{D} r d^{D} k\left(r^{2}\right)^{i}\left(k^{2}\right)^{j}\left(q^{2}\right)^{l}(p-q-r)^{2 m}(r-k)^{2 n}, \\
\mathcal{S}_{N D I M}^{5}=\int d^{D} q d^{D} r d^{D} k\left(q^{2}\right)^{i}\left(k^{2}\right)^{j}\left(r^{2}\right)^{l}(p-r)^{2 m}(r-q)^{2 n}(p-r-k)^{2 s}, \\
\mathcal{S}_{N D I M}^{6}=\int d^{D} q d^{D} r d^{D} k\left(r^{2}\right)^{i}\left(k^{2}\right)^{j}\left(q^{2}\right)^{l}(r+k)^{2 m}(p-k-r)^{2 n}(r+k-q)^{2 s},
\end{gathered}
$$

and

$$
\mathcal{S}_{N D I M}^{7}=\int d^{D} k d^{D} q d^{D} r\left(q^{2}\right)^{i}\left(k^{2}\right)^{j}(p-k-r)^{2 l}(k-q)^{2 m}(p-k)^{2 n}\left(r^{2}\right)^{s} .
$$

All these can be evaluated in a similar way following the steps of the previous section. For this reason we quote here only the final results, after continuation to positive dimension and negative exponents of propagators:

$$
\mathcal{S}_{N D I M}^{2}=\pi^{D}\left(k^{2}\right)^{\rho}\left\{\mathbf{C}_{3} F_{2}\left(a_{2}, b_{2}, c_{2} ; e_{2}, f_{2} \mid 1\right)+\mathbf{D}_{3} F_{2}\left(a_{2}^{\prime}, b_{2}^{\prime}, c_{2}^{\prime} ; e_{2}^{\prime}, f_{2}^{\prime} \mid 1\right)\right\}
$$

where

$$
\begin{aligned}
\mathbf{C} & \equiv(-i \mid \rho)(\rho+D / 2 \mid-2 \rho-D / 2)(i+D / 2-\rho \mid \rho)(\rho-i-l \mid-j-D / 2) \\
& \times(-j \mid-m-n-D / 2)(-n \mid j+m+n+D / 2)
\end{aligned}
$$

and

$$
\begin{aligned}
\mathbf{D} & \equiv(-i \mid \rho)(-l \mid \rho)(\rho+D / 2 \mid-2 \rho-D / 2)(j+m+n+D \mid-j-m-D / 2) \\
& \times(-j-m \mid-n-D / 2)(-n \mid j+m+n+D / 2),
\end{aligned}
$$

with the set of parameters defined by $\left(a_{2}, b_{2}, c_{2} ; e_{2}, f_{2}\right)=(-m, i+D / 2,-j-m-n-D / 2 ; i+D / 2-\rho, 1-m-n-D / 2)$ and $\left(a_{2}^{\prime}, b_{2}^{\prime}, c_{2}^{\prime} ; e_{2}^{\prime}, f_{2}^{\prime}\right)=(-j,-\rho,-j-m-n-D / 2 ; 1+l-\rho,-j-m)$ and $\rho=\sigma-s$. Note that here also we have a linear combination of two ${ }_{3} F_{2}$ hypergeometric functions, since $\left(e_{2}-f_{2}\right)$ is an integer and N $\phi$ rlund's theorem applies.

$$
\begin{aligned}
\mathcal{S}_{N D I M}^{3}= & \pi^{D}\left(k^{2}\right)^{\rho}(-l \mid \rho)(\rho+D / 2 \mid-2 \rho-D / 2)(i+l+D / 2-\rho \mid \rho)(-j \mid j+n+D / 2) \\
& \times(-n \mid j+n+D / 2)(j+n+D \mid-2 j-2 n-3 D / 2),
\end{aligned}
$$




$$
\begin{aligned}
\mathcal{S}_{N D I M}^{4}= & \pi^{3 D / 2}\left(p^{2}\right)^{\rho^{\prime}}(-j \mid j+n+D / 2)(-n \mid j+n+D / 2)(-l \mid l+m+D / 2) \\
& \times(-m \mid l+m+D / 2)\left(\rho^{\prime}+D / 2 \mid-2 \rho^{\prime}-D / 2\right)(j+n+D \mid i) \\
& \times(-j-n-D / 2 \mid-i),
\end{aligned}
$$

where $\rho^{\prime}=\rho+D / 2$.

$$
\begin{aligned}
\mathcal{S}_{N D I M}^{5}= & \pi^{3 D / 2}\left(p^{2}\right)^{\sigma^{\prime}}(-i \mid i+n+D / 2)(-n \mid i+n+D / 2)(-j \mid j+s+D / 2) \\
& \times(-s \mid j+s+D / 2)(-i-l-n-D / 2 \mid l)(-j-m-s-D / 2 \mid m) \\
& \times\left(\sigma^{\prime}+D / 2 \mid-2 \sigma^{\prime}-D / 2\right)(i+n+D \mid l)(j+s+D \mid m),
\end{aligned}
$$

where $\sigma^{\prime}=\sigma+D / 2$,

$$
\begin{aligned}
\mathcal{S}_{N D I M}^{6}= & \pi^{3 D / 2}\left(p^{2}\right)^{\sigma^{\prime}}(-i \mid i+j+D / 2)(-j \mid i+j+D / 2)(-l \mid l+s+D / 2)(-s \mid l+s+D / 2) \\
& \times\left(\sigma^{\prime}+D / 2 \mid-2 \sigma^{\prime}-D / 2\right)(-i-j-l-m-s-D \mid l+m+s+D / 2) \\
& \times(i+j+D \mid l+m+s+D / 2)(-n \mid-l-s-D / 2+n) \\
& \times(l+s+D \mid-l-s-D / 2+n) .
\end{aligned}
$$

Note that this result contains a special case (for $m=0$ ) calculated numerically in [16]. Our result, on the other hand, was analytically obtained and is more general, since the exponents of propagators are arbitrary (cf. results in the second reference in [5]).

Finally,

$$
\begin{aligned}
\mathcal{S}_{N D I M}^{7}= & \pi^{3 D / 2}\left(p^{2}\right)^{\sigma^{\prime}}(-i \mid i+m+D / 2)(-m \mid i+m+D / 2)(-l \mid l+s+D / 2) \\
& \times\left(\sigma^{\prime}+D / 2 \mid-2 \sigma^{\prime}-D / 2\right)(-i-j-m-D / 2 \mid j)(l+s+D \mid-2 l-2 s-3 D / 2) \\
& \times\left(-l-n-s-D / 2 \mid \sigma^{\prime}\right)(i+m+D \mid-i+l-m+n+s)(-s \mid l+s+D / 2) .
\end{aligned}
$$

\section{CONCLUSION.}

Although NDIM is not a regularization method, it shares all the concepts of dimensional regularization since it is based on the principle of analytic continuation in the dimension of space-time. In this work we showed that with an adequate technique such as NDIM it is possible to calculate some two- and three-loop Feynman integrals with relative easiness and without much elaborate machinery, just the performance of some Gaussian-type integrals plus resolution of systems of linear algebraic equations. Of course, in view of the great number of systems of linear algebraic equations that are involved, we need the help of computer facilities, but the task is simple, which a PC can handle properly.

The results we obtained can be used to study on-shell form factors in massless QCD at the two-loop level. Moreover, to illustrate how NDIM works beyond two-loops, we also performed some calculations of scalar three-loop, two-point integrals with arbitrary exponents for propagators and dimension, in Euclidean space.

\section{ACKNOWLEDGMENTS}

A.G.M.S. gratefully acknowledges FAPESP (Fundação de Amparo à Pesquisa do Estado de São Paulo, Brasil) for financial support.

[1] G.Kramer, B.Lampe, J.Math.Phys. 28 (1987) 945.

[2] R.J.Gonsalves, Phys.Rev. D 28 (1983) 1542. W.L. van Neerven, Nucl.Phys. B 268 (1986) 453.

[3] I.G.Halliday, R.M.Ricotta, Phys.Lett. B 193 (1987) 241. 
[4] G.V.Dunne, I.G.Halliday, Phys.Lett. B 193 (1987) 247.

[5] A.T.Suzuki, A.G.M.Schmidt, JHEP 09 (1997) 002. A.T.Suzuki, A.G.M.Schmidt, Eur.Phys.J. C5 (1998) 175. A.T.Suzuki, A.G.M.Schmidt, Phys.Rev. D58 (1998) 047701.

[6] A.T.Suzuki, A.G.M.Schmidt, J.Phys.A31 (1998) 8023.

[7] A.T.Suzuki, A.G.M.Schmidt, hep-th/9712104.

[8] A.T.Suzuki, A.G.M.Schmidt, R.Bentín, Nucl.Phys.B537 (1999) 549.

[9] K.G.Chetyrkin, A.L.Kataev, F.V.Tkachov, Nucl. Phys. B174 (1980) 345. K.G.Chetyrkin, F.V.Tkachov, Nucl.Phys. B192 (1981) 159.

[10] E.E.Boos, A.I.Davydychev, Theor. Math. Phys. 89 (1991) 1052. A.I.Davydychev, J.Math. Phys. 32 (1991) 1052; J.Math. Phys. 33 (1991) 358.

[11] A.I.Davydychev, P.Osland, Phys.Rev.D59 (1999) 014006.

[12] A.Erdélyi, W.Magnus, F.Oberhettinger and F.G.Tricomi, Higher Transcendental Functions (McGraw-Hill, 1953). I.S.Gradshteyn, I.M.Rhyzik, Table of Integrals, Series and Products (Academic Press, 1994).

[13] W.N.Bailey, Generalized Hypergeometric Series (Cambridge Univ. Press, 1935).

[14] Y.L.Luke, Special Functions and Their Approximations (Vol.I, Academic Press, 1969). L.J.Slater, Generalized Hypergeometric Functions, (Cambridge Univ. Press, 1966)

[15] N.E.N申rlund, Acta Math. 94 (1955) 289.

[16] R.Easther, G. Guralnik, S.Hahn, hep-ph/9903255. 

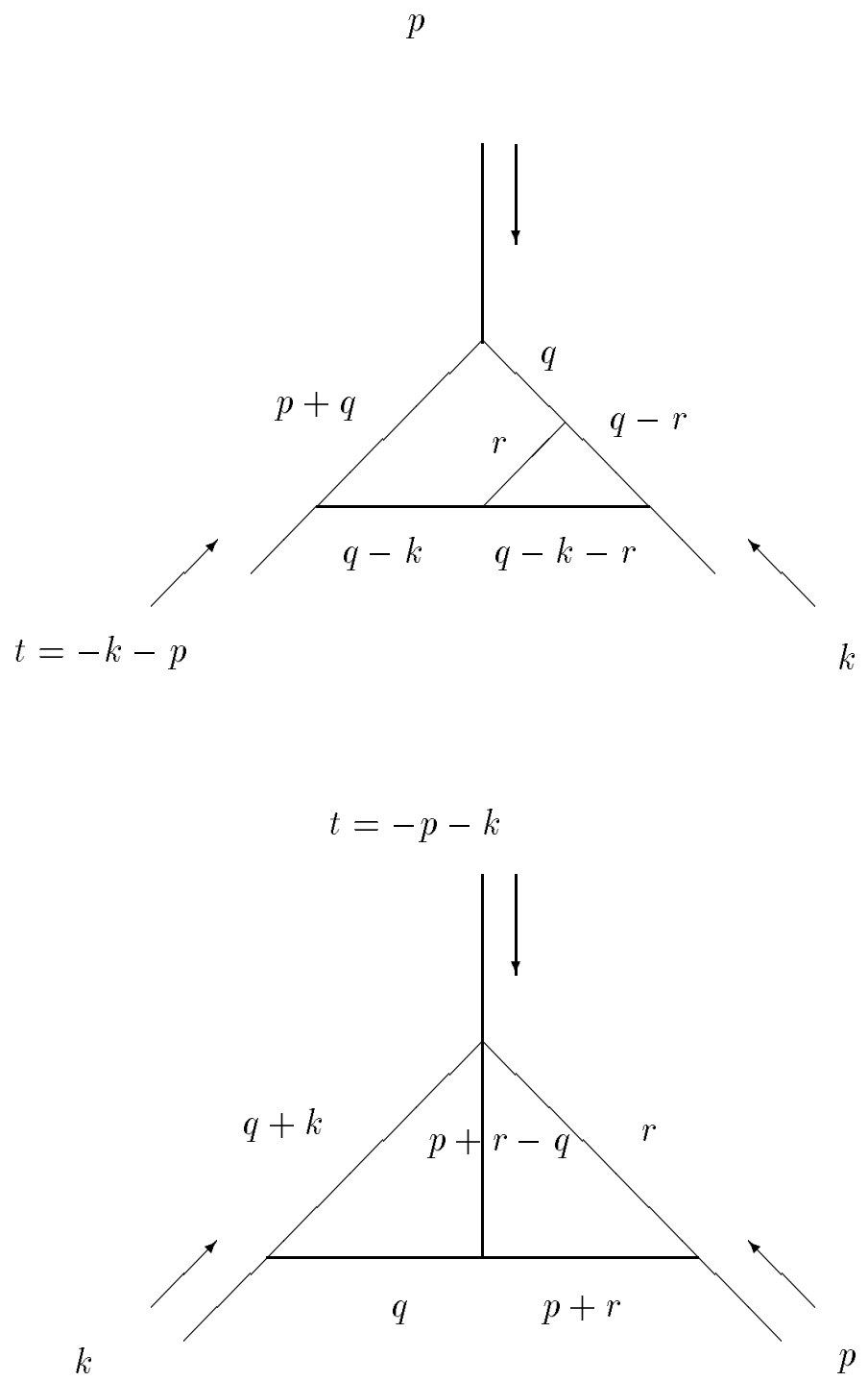


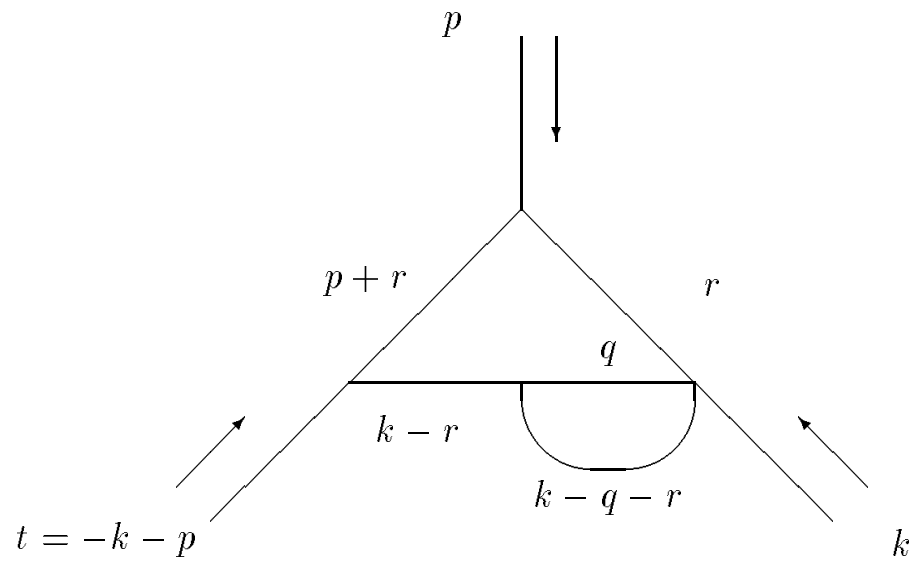




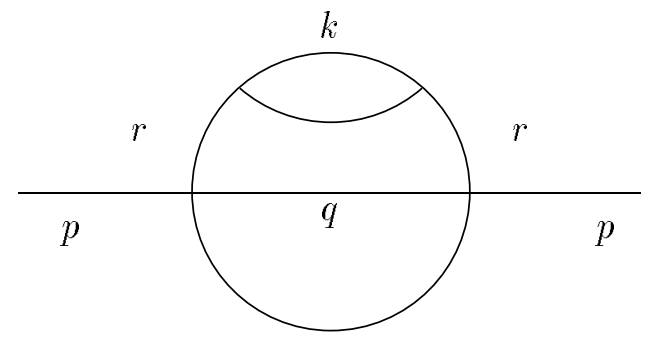




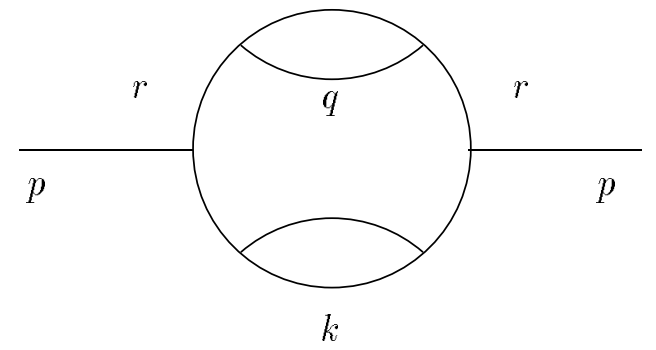




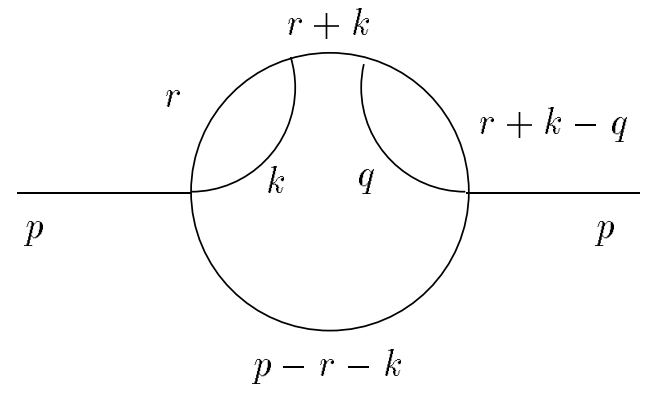




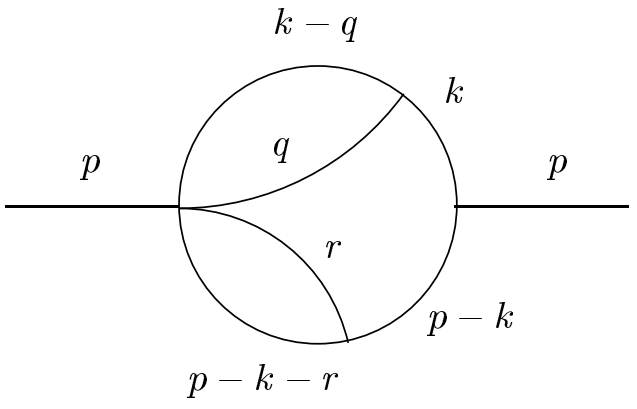

Figure 7. 